\title{
Impact of normothermic ex vivo lung perfusion on early post- transplantation cytomegalovirus infection
}

\author{
Achim Koch $^{1,2}$, Nikolaus Pizanis ${ }^{1,2}$, Vasiliki Bessa ${ }^{1,3}$, Alexis Slama ${ }^{1,4}$, Clemens Aigner $^{1,4}$, Christian Taube $^{1,3}$, \\ Markus Kamler ${ }^{1,2}$
}

${ }^{1}$ West German Center for Lung Transplantation, ${ }^{2}$ Department of Thoracic and Cardiovascular Surgery, ${ }^{3}$ Department of Pulmonology, ${ }^{4}$ Department of Thoracic Surgery and Surgical Endoscopy, University of Duisburg-Essen, Essen, Germany

Contributions: (I) Conception and design: A Koch, N Pizanis; (II) Administrative support: N Pizanis, V Bessa; (III) Provision of study patients: V Bessa, C Aigner, C Taube; (IV) Collection and assembly of data: A Koch, A Slama; (V) Data analysis and interpretation: A Koch, M Kamler; (VI) Manuscript writing: All authors; (VII) Final approval of manuscript: All authors.

Correspondence to: Achim Koch, MD, PhD. Department of Thoracic and Cardiovascular Surgery, University of Essen, Hufelandstrasse 55, 45147

Essen, Germany. Email: achim.koch@uk-essen.de.

Background: The low acceptance rates in lung transplantation underline the importance to use every potential transplantable organ. With the use of normothermic ex vivo lung perfusion (EVLP) there is a potential to use more donor lungs for transplantation. Aim of this study was to evaluate if EVLP has an effect on cytomegalovirus (CMV) infection after lung transplantation.

Methods: Between May 2016 and October 2018, 57 lung transplants were performed. Out of these 21 extended criteria lungs were evaluated by EVLP and 16 transplanted. In a retrospective study, results of EVLP treated lungs were compared with lungs after cold storage preservation (CSP). Donor/recipient CMV IgG status and seroconversion rate was examined.

Results: Donors were CMV IgG+ in EVLP 69\% and CSP 61\% (n.s.). Best $\mathrm{pO}_{2}$ on procurement at $\mathrm{FiO}_{2}$ 1.0 was in EVLP $278 \pm 76$ versus CSP $413 \pm 96 \mathrm{mmHg}(\mathrm{P} \leq 0.05)$. Recipients were CMV IgG+ in EVLP $38 \%$ and CSP 63\% $(\mathrm{P}<0.07)$. CMV seroconversion: EVLP 12\%, CSP 20\% $(\mathrm{P}<0.05)$, in the CSP group in $5 \%$ recipients with more than 1,000 copies/mL were diagnosed by PCR and treated for CMV infection. Procalcitonin (PCT) levels from day 1 to day 5 were significantly lower for CSP group $(\mathrm{P}<0.05)$. 30-day mortality was $12 \%$ for EVLP recipients.

Conclusions: Normothermic EVLP did not influence CMV infection rate, however early PCT levels were higher in EVLP group. Short-term results were comparable to standard lung transplantation.

Keywords: Cytomegalovirus infection (CMV infection); ex vivo lung perfusion (EVLP); extended criteria donors (ECD); lung transplantation

Submitted Oct 30, 2019. Accepted for publication Dec 17, 2019.

doi: $10.21037 /$ jtd.2020.02.26

View this article at: http://dx.doi.org/10.21037/jtd.2020.02.26

\section{Introduction}

Lung transplantation became a standard procedure in patients with end stage lung disease but despite optimized donor management only $40-50 \%$ of lungs from multiorgan-donors are transplanted. To overcome the shortness of donor organs, extended criteria donor (ECD) lungs are more frequently evaluated for transplantation by use of normothermic ex vivo lung perfusion (EVLP) (1). Previous studies showed that these lungs after pretransplant check can be transplanted with good results (2). Nakajima reported that EVLP treatment with broad spectrum antibiotics significantly reduced the bacterial burden and endotoxin levels in infected donor lungs (3). Despite antimicrobial and antiviral prophylaxis infections are major contributors to morbidity and mortality after 
lung transplantation. Especially cytomegalovirus (CMV) is a very common pathogen $(4,5)$. Little is known about the postoperative course of EVLP lungs and incidence of CMV infection.

It was the aim of this study assess if there was an influence of normothermic EVLP on early perioperative CVM conversion in comparison to a cohort of patients after standard cold storage preservation (CSP).

\section{Methods}

In a single center analysis, we retrospectively analyzed data of 57 lung transplant recipients transplanted between Mai 2016 and October 2018. The study was approved by the Local Ethics Committee of the University Duisburg-Essen (15-6711-BO) and conducted according to the declaration of Helsinki. Lung offers were allocated according to Eurotransplant allocation rules. All CMV donor+/ recipient+, CMV D-/R+ (intermediate risk for $\mathrm{CMV}$ reactivation) CMV D-/R- (low risk) and $\mathrm{D}+/ \mathrm{R}-$ (high risk) single and bilateral lung transplant (LT) recipients were included. Every lung offer was assessed by two experienced transplant surgeons. Lungs were either directly used for transplantation or categorized as ECD if at least one or more of the following criteria was met: $\mathrm{PaO}_{2} / \mathrm{FiO}_{2}<300$, age $>55$, signs of aspiration, smoking history, abnormalities on chest X-ray or evidence of contusion/trauma (6). These lungs were regarded as to be potentially salvable if: $\mathrm{PaO}_{2} /$ $\mathrm{FiO}_{2}<300$ without obvious explanation, (neurogenic-) lung edema, pulmonary infections, pulmonary embolism. With regard to these criteria 21 ECD donor lungs were assessed by EVLP. After 4-hour evaluation 5 donor lungs had to be declined, 16 lungs were transplanted. 41 lungs were transplanted with the use of CSP.

After acceptance donor lungs were evaluated in the donor hospital by bronchoscopy, direct inspection and for gas exchange function. All donors were treated according to a standard donor protocol with oro-tracheal suction, increased cuff pressure and antibiotic therapy. When last $\mathrm{PaO}_{2} / \mathrm{FiO}_{2}$ before organ retrieval was below 300 and the donor lungs were regarded potentially salvable according to the above mentioned criteria, the decision was made to use EVLP. For preservation all donor lungs were perfused with $4 \mathrm{~L}$ ( $3 \mathrm{~L}$ antegrade/1 $\mathrm{L}$ retrograde) low potassium dextran solution (Perfadex, Xvivo, Denver, CO, USA) at $4{ }^{\circ} \mathrm{C}$ and transported on ice. After transport EVLP lungs were mounted on the EVLP system (XPS, Xvivo, Denver, CO, USA). The technique of EVLP has been described in detail elsewhere $(7,8)$. After cold preservation donor lungs were cannulated via the pulmonary artery and the left atrium to the EVLP system. The EVLP system consisted of a perfusion circuit with a centrifugal pump, oxygenator, a heater-cooler, a ventilator and a monitoring console. Donor lungs were placed in a sterile dome and perfused with the albumin and dextran rich Steen-solution (Xvivo, Denver, CO, USA). During the 4-hour perfusion procedure lungs were gradually rewarmed and after reaching a temperature of $32{ }^{\circ} \mathrm{C}$ ventilation with a calculated tidal volume and PEEP of $5 \mathrm{~cm} \mathrm{H}_{2} \mathrm{O}$ is started. Perfusion volume was aimed at $40 \%$ cardiac-output. The oxygenator served as a de-oxygenator by administration of a venous gas mix $\left(6 \% \mathrm{O}_{2}, 8 \% \mathrm{CO}_{2}\right.$, $86 \%$ nitrogen). After every hour a recruitment maneuver with increased tidal volume was performed to evaluate the oxygenation capacity of the donor lungs. The difference of oxygen content between pulmonary venous and pulmonary arterial perfusion solution was calculated $\left(\Delta \mathrm{pO}_{2}\right)$. Lungs that were regarded transplantable, were expected to archive a $\Delta \mathrm{pO}_{2}>350 \mathrm{mmHg}$ at the end of the 4-hours process.

\section{Clinical follow-up}

\section{Immunosuppressive regimen}

T-lymphocyte guided antithymocyte globulin (ATG Tecelac) $1 \mathrm{mg} / \mathrm{kg}$ body weight was administered as induction therapy. For maintenance immunosuppression patients received a calcineurin inhibitor (tacrolimus) and a proliferation inhibitor (mycophenolate mofetil and prednisone). Routine bronchoscopies were performed on postoperative day one, before weaning from ventilator and weekly thereafter. In clinical suspicion of an acute cellular rejection we performed cortison pulse therapy with $10 \mathrm{mg} / \mathrm{kg}$ body weight methylprednisolone intravenously for 3 days.

\section{Antimicrobial prophylaxis}

Lung transplant recipients received a routine perioperative antibiotic prophylaxis with piperacillin/ tazobactam. Antibiotic prophylaxis was maintained for five postoperative days. On postoperative day 3, pneumocystis jirovecii prophylaxis was started with trimethoprimsulfamethoxazole indefinitely. For antifungal prophylaxis inhalative amphotericin B was given. Independent of the CMV status all patients received a renally adjusted antiviral prophylaxis for 7 days using ganciclovir $(5 \mathrm{mg} / \mathrm{kg}$ twice a day) combined with CMV hyperimmunoglobulin $(100 \mathrm{IU} / \mathrm{mL} \cdot \mathrm{kg})$ intravenously once weekly for four weeks. The antiviral prophylaxis was maintained in all lung 
transplant recipients for at least one year.

\section{Clinical variables}

Demographic and clinical data were retrospectively collected from the patients and electronically stored in medical records. Transplant relevant information as age, sex, indication for transplantation, ventilation time and length of ICU stay and in-hospital stay, as well as immunosuppression, antiviral prophylaxis and episodes of CMV infections were included. C-reactive protein (CRP) and procalcitonin (PCT) values were reviewed in the early postoperative period (5 postoperative days). $\mathrm{CMV}$ infection and disease were classified according to the American Society of Transplantation recommendations and the international consensus guidelines (9). Asymptomatic CMV infection was defined as the detection of CMV $(>1,000$ copies $/ \mathrm{mL}$ in bronchoalveolar lavage fluid or $>500$ copies/mL in blood) without clinical manifestations. In contrast CMV disease was defined as presence of CMV in blood, bronchoalveolar lavage fluid and tissue plus clinical symptoms like viral syndrome manifestations.

\section{Statistical analysis}

SPSS Version 21.0 (IBM Corp., SPSS Statistics, Armonk, NY, USA), was used for statistical analysis. Continuous variables are summarized as mean (standard deviation), unless indicated otherwise and categorical variables as counts (percentages). Continuous data were evaluated for normality of distribution using the Shapiro-Wilk's test. Two-sided $t$-test was used for comparison of continuous, normally distributed data. The Chi-square test was used for testing association between two categorical variables. The level of significance was set to $<0.05$.

\section{Results}

\section{Patient population}

In this study, the CMV status in the early postoperative period was evaluated for 57 lung transplant recipients. In the normothermic EVLP group 21 lungs were evaluated. Out of these, 16 lungs were found to be successfully transplantable, 5 organs had to be discarded. All 41 lung offers in the CSP group were transplanted. In the EVLP group there were 14 bilateral, 1 unilateral and 1 bilobar lung transplantation, in the CSP group all 41 lung transplants were bilateral. The demographic and clinical data for donors and recipients are shown in Tables 1 and 2. Most common indication for lung transplantation was COPD including alpha-1 antitrypsin deficiency followed by idiopathic pulmonary fibrosis. Concerning the demographic data for both groups no significant differences were observed. At the time of procurement significant differences in the EVLP group compared with the CSP group were found: In the EVLP group gas exchange function was significantly worse (best donor P/F $278 \pm 76$ vs. $413 \pm$ $96 \mathrm{mmHg}$ in CSP; $\mathrm{P} \leq 0.05)$. Compared to the $\mathrm{P} / \mathrm{F}$ ratio of the donors in the EVLP group $\mathrm{P} / \mathrm{F}$ ratio improved significantly from hour 1 to $3(354 \pm 86,364 \pm 59$ and $401 \pm 34 \mathrm{mmHg}, \mathrm{P}<0.05)$. In the EVLP group we found two patients $(14 \%)$ with primary graft dysfunction grade 3 at 72 hours $(\mathrm{P}<0.05)$.

\section{CMV infection and disease}

Donors were CMV IgG+ in EVLP 69\% and CSP 61\% (n.s.). Recipients were CMV IgG+ in EVLP $38 \%$ and CSP $63 \%(\mathrm{P}<0.07)$. The seroconversion rate in the EVLP group (12\%) showed a trend to be lower compared to the CSP (20\%) group. All patients received an antiviral prophylaxis with (val-) ganciclovir for a minimum of 1 year. No symptomatic CMV disease was observed during the first 30 postoperative days (Table 3).

Procalcitonin (PCT) levels $(\mathrm{ng} / \mathrm{mL})$ on the first five postoperative days were significantly lower in the CSP group (Table 4).

\section{Acute rejection episodes}

During the first 30 days, a limited number of low-grade (A1) acute cellular rejection (EVLP group 1 instance, CSP group 3 instances, n.s.) episodes were observed. All acute cellular rejection episodes resolved with corticosteroid pulse therapy. Three months postoperatively, in the CSP group two patients with clinical signs of acute antibody-mediated rejection and development of de novo donor specific antibodies were successfully treated by plasmapheresis.

\section{Mortality outcome}

The 30 -day mortality was $12 \%$ for EVLP recipients. One year survival rates were not significantly different $(95 \%$ in CSP group and 78\% in EVLP group). 
Table 1 Clinical and demographic characteristics of 57 lung transplant recipients

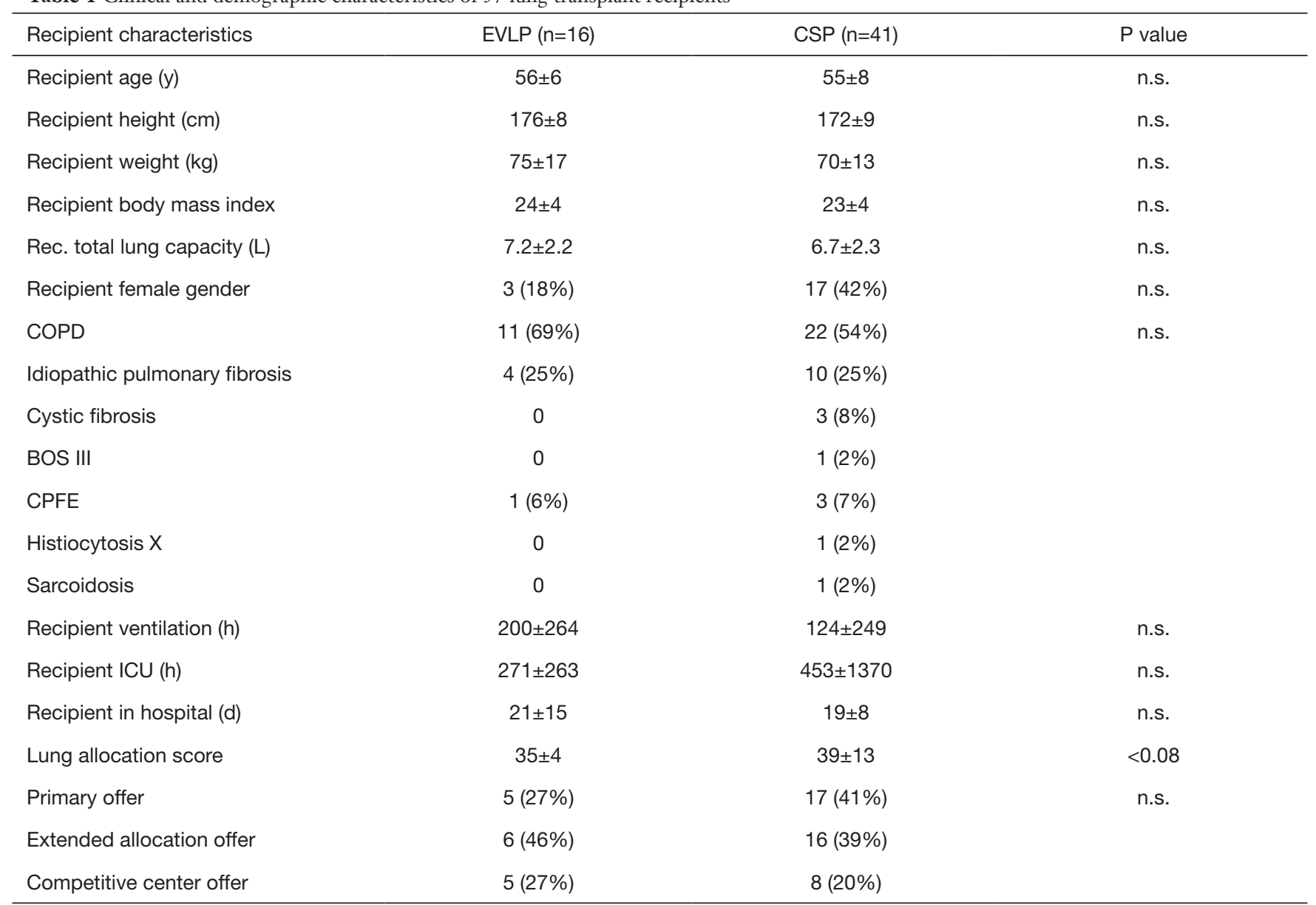

EVLP, ex vivo lung perfusion; CSP, cold storage preservation.

\section{Discussion}

This study demonstrated that normothermic EVLP is a safe tool for evaluation of donor lungs previously deemed not transplantable. In addition, this study showed that EVLP treatment did not negatively affect the post-transplantation CMV seroconversion rate in a cohort of 57 lung transplant recipients.

The discrepancy between demand for lung transplantation and availability of transplantable lung offers is a growing problem in recent years (10). Despite of extension of donor lung criteria, acceptance rates for donor organs in LTx remain the lowest among solid organ transplantation. Donor related risk factors for primary graft dysfunction are not fully understood adherence to standard donor criteria leads to discard of lungs that might be usable after assessment of graft function by means of EVLP (11). Normothermic EVLP is used to achieve a "more complete" and objective evaluation of lung function with the potential option to "repair" injured donor lungs (12).

In this retrospective study, we analyzed our experience with normothermic-EVLP and evaluated an influence of CMV seroconversion in the early postoperative period. CMV disease rates are higher in LTx recipients than in all other solid organ transplants. In absence of preventive therapy CMV causes infection in up to $80 \%$ of LTx recipients and is a major contributor to morbidity and mortality after LTx. Its indirect effects include increased risk for other infections and frequent rejection episodes leading to chronic lung allograft dysfunction (4). The CMV virus has a predilection for lung parenchyma and latent viral loads and seroconversion rates above $20 \%$ are reported in the literature. The lower CMV seroconversion rates observed in our study may be attributed to a reduction of viral load by dilution during perfusion in EVLP. The greatest risk of 
Table 2 Clinical and demographic characteristics of 57 lung transplant donors

\begin{tabular}{|c|c|c|c|}
\hline Donor characteristics & $\operatorname{EVLP}(n=16)$ & $\operatorname{CSP}(n=41)$ & $P$ value \\
\hline Donor height (cm) & $178 \pm 10$ & $173 \pm 9$ & $<0.05$ \\
\hline Donor weight (kg) & $98 \pm 22$ & $78 \pm 18$ & $<0.05$ \\
\hline Donor TLC (L) & $6.8 \pm 1.1$ & $6.2 \pm 1.1$ & n.s. \\
\hline Female donors & $5(31 \%)$ & $20(49 \%)$ & n.s. \\
\hline Best $\mathrm{PO}_{2}$ retrieval $(\mathrm{mmHg})$ & $278 \pm 76$ & $413 \pm 96$ & $\leq 0.05$ \\
\hline $\mathrm{pO}_{2} 1^{\text {st }}$ hour $(\mathrm{mmHg})$ & $354 \pm 86$ & & \\
\hline $\mathrm{pO}_{2} 2^{\text {nd }}$ hour $(\mathrm{mmHg})$ & $364 \pm 59$ & & \\
\hline Cerebrovasc. accident & $2(13 \%)$ & $8(20 \%)$ & \\
\hline Hanging & $3(19 \%)$ & 0 & \\
\hline Hypoxia & $3(19 \%)$ & $3(7 \%)$ & \\
\hline Intracranial bleed & $5(31 \%)$ & $19(46 \%)$ & \\
\hline Meningitis & $1(6 \%)$ & $2(5 \%)$ & \\
\hline Trauma & $2(13 \%)$ & $9(22 \%)$ & \\
\hline Mean out of body time (min) & $664 \pm 155$ & $333 \pm 77$ & $\leq 0.05$ \\
\hline
\end{tabular}

EVLP, ex vivo lung perfusion; CSP, cold storage preservation.

Table 3 Cytomegalovirus seroconversion rate (donor-D, recipient-R)

\begin{tabular}{|c|c|c|c|}
\hline Variable & $\operatorname{EVLP}(n=16)$ & $\operatorname{CSP}(n=41)$ & $P$ value \\
\hline CMV D+/R- & $6(38 \%)$ & $10(24 \%)$ & n.s. \\
\hline CMV D+/R+ & $5(31 \%)$ & $15(37 \%)$ & \\
\hline CMV D-/R- & $4(25 \%)$ & $4(10 \%)$ & \\
\hline High risk & $6(38 \%)$ & $10(24 \%)$ & n.s. \\
\hline Intermediate risk & $6(38 \%)$ & $24(59 \%)$ & \\
\hline Low risk & $5(31 \%)$ & 7 (17\%) & \\
\hline
\end{tabular}

EVLP, ex vivo lung perfusion; CSP, cold storage preservation; CMV, cytomegalovirus.

CMV disease is among seronegative recipients (R-) who received organs from seropositive donors (D+). Thus, the use of EVLP can serve as an additional preventive strategy apart from ganciclovir regimes which effectively reduce
CMV infections. However, use of intravenous ganciclovir is limited by systemic toxicity and oral ganciclovir has limited bioavailability and resorption. As CMV infections are most common within the first postoperative year, guidelines 
Table 4 Procalcitonin (PCT) and C-reactive protein (CRP) levels on postoperative day 1 to day 5

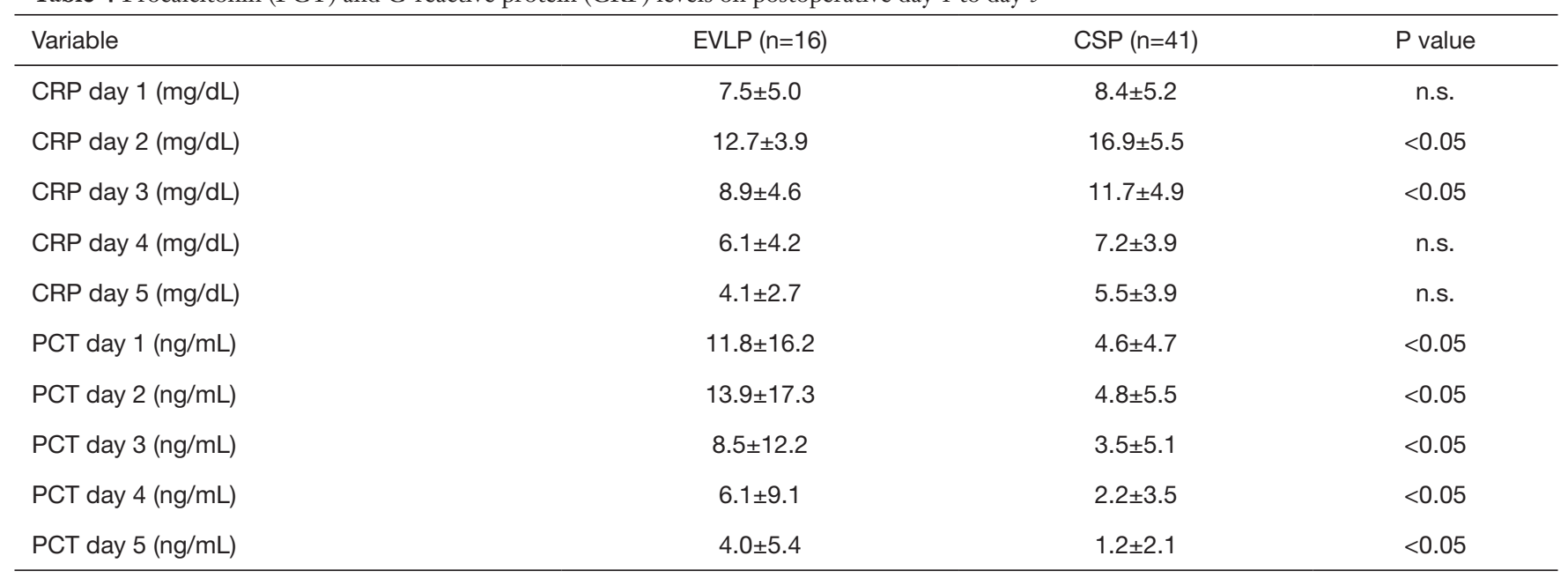

EVLP, ex vivo lung perfusion; CSP, cold storage preservation.

recommend 6-12 months of prophylaxis in $\mathrm{D}+\mathrm{R}$ - patients and at least six months among CMV R+ recipients. Treatment is recommended for any level of CMV replication (whole blood PCR). Direct effects of CMV are fever, bone marrow suppression and tissue invasive disease, including pneumonia. $\mathrm{CMV}$ infections and disease occurred after discontinuation of antiviral prophylaxis that was necessary for leukopenia caused by valganciclovir related myelosuppression. In valganciclovir resistant CMV disease mortality is up to $100 \%$, it remains a major clinical problem among $\mathrm{D}+/ \mathrm{R}-\mathrm{LuTx}$ recipients despite valganciclovir prophylaxis.

For evaluation by EVLP we included donor lungs with impaired gas exchange $\left(\mathrm{PaO}_{2}<350 \mathrm{mmHg}\right)$, lungs with pulmonary edema, suspect of aspiration or bilateral infiltrates on chest X-ray. Although EVLP is mainly used in donation after cardiac death, our donors were brain death. The EVLP group had a mean out-of-body time of $664 \pm 128 \mathrm{~min}$, which was significantly longer $(\mathrm{P}<0.05)$ than the average ischemic time (333 $\pm 76 \mathrm{~min})$ of the CSP group. Prolonged ischemic (outof-body) times may have an impact on transplant outcome. However, duration of EVLP does not contribute to ischemic time as lungs are oxygenated and perfused with nutrients (13). No adverse outcome in the EVLP group was observed.

We found no significant differences for the postoperative mechanical ventilation time, length of ICU-stay or hospitalization between EVLP and non-EVLP transplants. So far, we did not observe anastomotic complications. The perioperative 30-day survival rates were excellent for both groups (12\% vs. $2 \%, \mathrm{P}=$ n.s.). Two patients died in the EVLP group due to multi organ failure after 1 and 37 days, the causes of death could not be attributed to the use of EVLP. These results are in concordance with other groups where EVLP survival rates compared to conventional lung recipients were significantly better and early and midterm results were not negatively affected by the use of EVLP (14).

Other positive aspects of EVLP are: donor leukocytes depleted during EVLP might reduce direct allorecognition and T-cell priming and therefore diminishes recipient T-cell infiltration into the graft. EVLP can be used to remove donor leukocytes, possibly a novel approach to tackle acute rejection (15). The higher PCT levels in the EVLP group may be reactive to cytokines released during the EVLP process.

\section{Limitations}

The retrospective character and the small patient number especially in the EVLP group is a major limitation of this study. Furthermore the follow-up time is limited to the early postoperative period. In addition, the administration of CMV immunoglobulin post-transplantation may also lead to CMV sero-conversation, which should be considered as a possible confounding factor in our study. However, the influence of EVLP on post-transplant infectious situation and immunological impact is a field that needs to be further highlighted. This study can be seen as a first approach to this fascinating opportunity in transplantation.

\section{Conclusions}

Normothermic EVLP can safely be used in the evaluation 
of lungs initially considered unacceptable and did not negatively influence the CMV infection rate. Short term results are comparable to standard lung transplantation. This experience from a single center shows that transplantation of $76 \%$ of ECD lungs was possible.

\section{Acknowledgments}

Funding: None.

\section{Footnote}

Conflicts of Interest: All authors have completed the ICMJE uniform disclosure form (available at http://dx.doi. org/10.21037/jtd.2020.02.26). The authors have no conflicts of interest to declare.

Ethical Statement: The authors are accountable for all aspects of the work in ensuring that questions related to the accuracy or integrity of any part of the work are appropriately investigated and resolved. The study was approved by the Local Ethics Committee of the University Duisburg-Essen (15-6711-BO).

Open Access Statement: This is an Open Access article distributed in accordance with the Creative Commons Attribution-NonCommercial-NoDerivs 4.0 International License (CC BY-NC-ND 4.0), which permits the noncommercial replication and distribution of the article with the strict proviso that no changes or edits are made and the original work is properly cited (including links to both the formal publication through the relevant DOI and the license). See: https://creativecommons.org/licenses/by-nc-nd/4.0/.

\section{References}

1. Cypel M, Keshavjee S. Extending the donor pool: rehabilitation of poor organs. Thorac Surg Clin 2015;25:27-33.

2. Slama A, Schillab L, Barta M, et al. Standard donor lung procurement with normothermic ex vivo lung perfusion: A prospective randomized clinical trial. J Heart Lung Transplant 2017;36:744-53.

3. Nakajima D, Cypel M, Bonato R, et al. Ex Vivo Perfusion Treatment of Infection in Human Donor Lungs. Am J Transplant 2016;16:1229-37.

4. Beam E, Lesnick T, Kremers W, et al. Cytomegalovirus disease is associated with higher all-cause mortality after lung transplantation despite extended antiviral prophylaxis. Clin Transplant 2016;30:270-8.

5. Natori Y, Humar A, Husain S, et al. Recurrence of CMV Infection and the Effect of Prolonged Antivirals in Organ Transplant Recipients. Transplantation 2017;101:1449-54.

6. Martens A, Van Raemdonck DE, Smits J, et al. A retrospective database analysis to evaluate the potential of ex vivo lung perfusion to recruit declined lung donors. Transpl Int 2017;30:1002-10.

7. Cypel M, Keshavjee S. Extracorporeal lung perfusion. Curr Opin Organ Transplant 2011;16:469-75.

8. Koch A, Pizanis N, Olbertz C, et al. One-year experience with ex vivo lung perfusion: Preliminary results from a single center. Int J Artif Organs 2018;41:460-6.

9. Kotton CN, Kumar D, Caliendo AM, et al. Updated international consensus guidelines on the management of cytomegalovirus in solid-organ transplantation. Transplantation 2013;96:333-60.

10. Chambers DC, Yusen RD, Cherikh WS, et al. The Registry of the International Society for Heart and Lung Transplantation: Thirty-fourth Adult Lung And HeartLung Transplantation Report-2017; Focus Theme: Allograft ischemic time. J Heart Lung Transplant 2017;36:1047-59.

11. Aigner C, Slama A, Hotzenecker K, et al. Clinical ex vivo lung perfusion--pushing the limits. Am J Transplant 2012;12:1839-47.

12. Koch A, Olbertz, C, Abou-Issa, O, et al. Ex-vivoLungenperfusion. Ein Werkzeug zur optimierten Nutzung von Spenderorganen. ZHTG 2017. doi: 10.1007/s00398017-0175-9.

13. Tikkanen JM, Cypel M, Machuca TN, et al. Functional outcomes and quality of life after normothermic ex vivo lung perfusion lung transplantation. J Heart Lung Transplant 2015;34:547-56.

14. Cypel M, Yeung JC, Machuca T, et al. Experience with the first 50 ex vivo lung perfusions in clinical transplantation. J Thorac Cardiovasc Surg 2012;144:1200-6.

15. Stone JP, Critchley WR, Major T, et al. Altered Immunogenicity of Donor Lungs via Removal of Passenger Leukocytes Using Ex Vivo Lung Perfusion. Am J Transplant 2016;16:33-43.

Cite this article as: Koch A, Pizanis N, Bessa V, Slama A, Aigner C, Taube C, Kamler M. Impact of normothermic ex vivo lung perfusion on early post-transplantation cytomegalovirus infection. J Thorac Dis 2020;12(4):1350-1356. doi: 10.21037/ jtd.2020.02.26 